\title{
Dynamic Behavior Analysis of Touchdown Process in Active Magnetic Bearing System Based on a Machine Learning Method
}

\author{
Zhe Sun, ${ }^{1,2,3}$ Xunshi Yan, ${ }^{1,2,3}$ Jingjing Zhao, ${ }^{1,2,3}$ Xiao Kang, \\ Guojun Yang, ${ }^{1,2,3}$ and Zhengang Shi ${ }^{1,2,3}$ \\ ${ }^{1}$ Institute of Nuclear and New Energy Technology, Tsinghua University, Beijing 100084, China
${ }^{2}$ Collaborative Innovation Center of Advanced Nuclear Energy Technology, Beijing 100084, China
${ }^{3}$ The Key Laboratory of Advanced Reactor Engineering and Safety, Ministry of Education, Beijing 100084, China
${ }^{4}$ Mechanical Engineering, Texas A\&M University, College Station, TX 77843, USA \\ Correspondence should be addressed to Zhe Sun; sun_zhe@tsinghua.edu.cn
}

Received 30 December 2016; Revised 28 August 2017; Accepted 14 September 2017; Published 18 October 2017

Academic Editor: Eugenijus Ušpuras

Copyright (c) 2017 Zhe Sun et al. This is an open access article distributed under the Creative Commons Attribution License, which permits unrestricted use, distribution, and reproduction in any medium, provided the original work is properly cited.

\begin{abstract}
Magnetic bearings are widely applied in High Temperature Gas-cooled Reactor (HTGR) and auxiliary bearings are important backup and safety components in AMB systems. The performance of auxiliary bearings significantly affects the reliability, safety, and serviceability of the AMB system, the rotating equipment, and the whole reactor. Research on the dynamic behavior during the touchdown process is crucial for analyzing the severity of the touchdown. In this paper, a data-based dynamic analysis method of the touchdown process is proposed. The dynamic model of the touchdown process is firstly established. In this model, some specific mechanical parameters are regarded as functions of deformation of auxiliary bearing and velocity of rotor firstly; furthermore, a machine learning method is utilized to model these function relationships. Based on the dynamic model and the Kalman filtering technique, the proposed method can offer estimation of the rotor motion state from noisy observations. In addition, the estimation precision is significantly improved compared with the method without learning. The proposed method is validated by the experimental data from touchdown experiments.
\end{abstract}

\section{Introduction}

Magnetic bearings are widely applied in High Temperature Gas-cooled Reactor (HTGR), where the rotating machines are running under highly purified helium environment. Compared with conventional bearings, Active Magnetic Bearings (AMBs) possess several attractive advantages, such as no friction, no need of lubrication, and the ability of longterm high speed running. Some types of essential rotating equipment in HTGR are supported by AMBs, that is, the main helium circulator and the helium blower in the fuel circulation system. The reliability of this equipment, especially in accident condition, plays a crucial role in the safety of the whole nuclear plant.

In an AMB system, the rotating rotor is suspended by electromagnetic forces and there is no contact between the rotor and the stator. An AMB system is a complex mechatronic system and consists of a large number of structural, electronic components and sophisticated control software. AMB systems are usually equipped with a series of control, protection, backup, and safety components and software to ensure the reliability and the safety of the whole rotating equipment. The control and protection software of an AMB system is designed to suspend the rotor within a wide range of operating conditions. Some abnormal operation conditions, for example, long-term overload, unexpected impact, and slight failure of components, are sustainable and can hardly affect the suspension. However, in the worst case, the magnetic suspension will fail and the rotor will touch down. To avoid damage to the rotor and the stator during the touchdown process, the so-called auxiliary bearings are necessary for AMB systems. The auxiliary bearings bear the rotating 
rotor during the touchdown process. They are important backup and safety components in AMB systems and can be regarded as the "last stand" for the safety of an AMB system.

In the applications associated with HTGR, the performance of auxiliary bearings significantly affects the reliability, safety, and serviceability of the AMB system, the rotating equipment, and the whole reactor. Research on the dynamic behavior during the touchdown process is crucial for analyzing the severity of the touchdown. Evaluating the contact forces between the rotor and the auxiliary bearings plays a central role of dynamic analysis of a touchdown process. However, due to the restrictions in structure design, it is difficult to equip force sensors in engineering rotating equipment. Thus these forces can hardly be measured directly and should be estimated from the acceleration and velocity of the rotor. On the other hand, only the displacements of the rotor are recorded in AMB systems. As is well known, estimating the velocity and acceleration from noisy displacement data is hardly realizable. Therefore this paper focuses on the estimation of velocities and accelerations of the rotor based on the dynamic model and the measured data.

Many remarkable achievements have been accomplished in the literature to estimate the highly nonlinear dynamic process associated with rotor touchdown. In 1991, Ishii and Kirk [1] built the touchdown model of the Jeffcott rotor, gained the transient response of the touchdown process by a numerical method, and obtained the optimal damping to reduce the displacement and the contact force. In 1994, Fumagali and Schweitzer [2] analyzed the influence of the air gap, friction coefficient, supporting damping, and stiffness on the dynamic behavior of rotors touchdown process. Fang and Barrett [3] added the Coulomb friction to Kirks mathematical model and gained the transient response using the Newmark$\beta$ method. In 2003, Zeng [4] studied the dynamic behavior of the rotor drop onto the auxiliary bearing with different supporting conditions, found out the critical friction coefficient causing the backward whirling, and claimed the low friction coefficient and soft support can reduce the contact force and the possibility of occurring backward whirl. Sun et al. [57] considered rotor drop simulation of a flywheel energy storage system, numerically and experimentally. The results suggested therefore that the life of the auxiliary bearing can be extended by reducing auxiliary bearing clearance, dropping velocity, contact friction, and support stiffness, applying static side-loads and increasing support. Keogh and Yong [8] indicated that the rotor drop procedure can be treated as a combination of bounce and rub. They simulated transient thermal response for a series of contact conditions. Moreover, an active recovery strategy was also obtained to make the rotor return from a persistent contact state back to a contact-free state [9]. The majority of reported research involves horizontal rotor drop. However, in vertical rotor drop tests forward whirl is prevalent. In 2008, Tan et al. [10] built a finite element model of vertical arranged rotor drop onto the auxiliary bearings and got the equivalent stress distribution of the rotor and auxiliary bearings. In 2011, Zhao et al. [11] used ABAQUS to analyze stress and deformation in an auxiliary bearing. However, this paper lacks consideration of the dynamic behavior of the rotor. In 2013, Xiao et al. [12] analyzed the load characteristics of the ceramic ball auxiliary bearing and established a finite element model in ANSYS applied in the transient response of the dropped rotor. However, this work did not consider the friction between the rotor and auxiliary bearing. Sun et al. [13] estimated the displacements, velocities, and accelerations of the rotor during touchdown from both the dynamic model and noisy data, the dynamic model of the rotor is introduced, and Kalman filtering technique is applied. Most papers in this region emphasize the dynamic behavior of the rotor touchdown process and the influence of different factors such as the air gap, rotational speed, and the eccentricity. Only a few works pay attention to the process of data acquired by the experiments.

On the other hand, rapidly developing machine learning techniques offer an attractive solution for data process. In the region of motion control of mechatronic systems, soft sensing [14-16] is an important application of machine learning technique. Soft sensing is an approach to estimate hard-tomeasure variables of a dynamic system from easy-to-measure variables. The soft sensing technique can also be applied to improve the measurement quality of some variables by incorporating information from various sources. Many achievements of soft sensing based on Neural Network (NN) [17-21] and Support Vector Regression (SVR) [22, 23] are reported. These applications of soft sensing technique are closely related to the motion data process. However, to our best knowledge, no achievement of learning-based data process in the region of touchdown process is published.

In this paper, a novel touchdown process analysis method based on prior knowledge based learning technique is proposed. Similar to some above-mentioned literature, the presented work mainly utilizes a data-based design verification strategy. In other words, the proposed method depends on the actual data and will be implemented in the machine which is already designed and built and when touchdown incident or test has occurred.

More specifically, this paper is an extension of [13] and the dynamical model proposed in [13] is utilized. In [13], some mechanical parameters, such as stiffness, damping ration, and friction coefficient, are introduced and regarded as known constants. In this paper, however, these parameters are modeled as functions of deformation of auxiliary bearing and velocity of the rotor. These functions are mathematically modeled by a prior knowledge based machine learning method. Kalman filtering technique is then applied to estimate the velocity and accelerator of the rotor. The learningbased model is trained by minimizing the error between measured and estimated motion states of the rotor. The proposed method is validated by the results of touchdown experiments.

The proposed method can be utilized to evaluate the operational state and residual life of the auxiliary bearings [7, 24] after touchdown. The improvement in designing auxiliary bearing can be expected thereby.

\section{An Introduction to Prior Knowledge Based Kernel Regression}

Prior Knowledge Based Kernel Regression (PKBKR) [25] is an extension of SVR $[26,27]$. Similar to SVR, PKBKR 
is a sample-based modeling method. When some inputoutput relationship has to be modeled, some training samples (input-output pairs $(\mathbf{s}, \zeta)$ ) should be observed firstly, and then the model can be established. The process of establishing the model based on the training samples is usually called "training." PKBKR is also a kernel method; that is, a kernel function is applied to evaluate the relationship between various samples. A kernel function $Q: \mathbb{R}^{n} \times \mathbb{R}^{n} \mapsto \mathbb{R}$ is a bivariable real-valued function defined by the user. In this paper we use the notation $Q$ rather than conventional $K$ to avoid confusing with stiffness. For a sample $s$ and a sample set $T=\left\{\mathbf{t}_{1}, \ldots, \mathbf{t}_{m}\right\}$, we use the following notation:

$$
Q(\mathbf{s}, T)=\mathbf{Q}_{\mathbf{s}, T}=\left[\begin{array}{llll}
Q\left(\mathbf{s}, \mathbf{t}_{1}\right) & \cdots & Q\left(\mathbf{s}, \mathbf{t}_{m}\right)
\end{array}\right] .
$$

Suppose that $m$ samples $T=\left\{\mathbf{t}_{k}, \zeta_{k}\right\}_{k=1}^{m}$ are obtained; the PKBKR model is in the following form:

$$
\widetilde{\zeta}=\sum_{k=1}^{m} \alpha_{k} Q\left(\mathbf{t}_{k}, \mathbf{s}\right)=\mathbf{Q}_{\mathbf{s}, T} \boldsymbol{\alpha},
$$

where $\alpha_{k}$ is coefficients, $\boldsymbol{\alpha}=\left[\alpha_{1}, \ldots, \alpha_{m}\right]^{\top}$. The parameter $\alpha_{k}$ will be determined in the training procedure. The main difference between PKBKR and standard SVR is that the PKBKR algorithm allows introducing an initial guess $f_{0}$ of the known model. The regularization term in SVR, which is introduced to control the model complexity, is replaced by the distance between the estimated model and the initial guess in the function space. Compared with standard SVR, the incorporation of initial guess may produce a more reasonable model. The detailed implementation of PKBKR can be found in [25] and is ignored here due to the limitation of space.

In this paper, the output $\zeta$ is physically a mechanical quantity and cannot be measured directly, thus we use a function of $\zeta$ to train the model. Because of this and computational considerations, model (2) is slightly modified in this paper. The following model is used:

$$
\widetilde{\zeta}=\mathbf{Q}_{\mathbf{s}, T^{\prime}} \boldsymbol{\alpha},
$$

where $T^{\prime}$ is a sample set defined by the user and can be differed from the observed sample set $T$. The particularity in modeling and training of PKBKR models will be discussed in the following sections.

\section{Dynamic Model of Touchdown Process}

3.1. Basic Assumptions of Model. In this paper, auxiliary bearing in the form of angular contact rolling-element bearing and vertically arranged AMB system is studied. A bearing of this type can bear both radial and axial load. In other words, it can be utilized as a radial-axial auxiliary bearing.

The touchdown process is quite complicated, which involves highly nonlinear and coupled interactions between the rotor, inner ring, balls, and outer ring of auxiliary bearings. Among these interactions, the impact between the rotor and the inner ring of auxiliary bearing plays a dominant role. Thus, for simplicity, in this paper the interaction between the rotor and a radial-axial auxiliary bearing is considered,

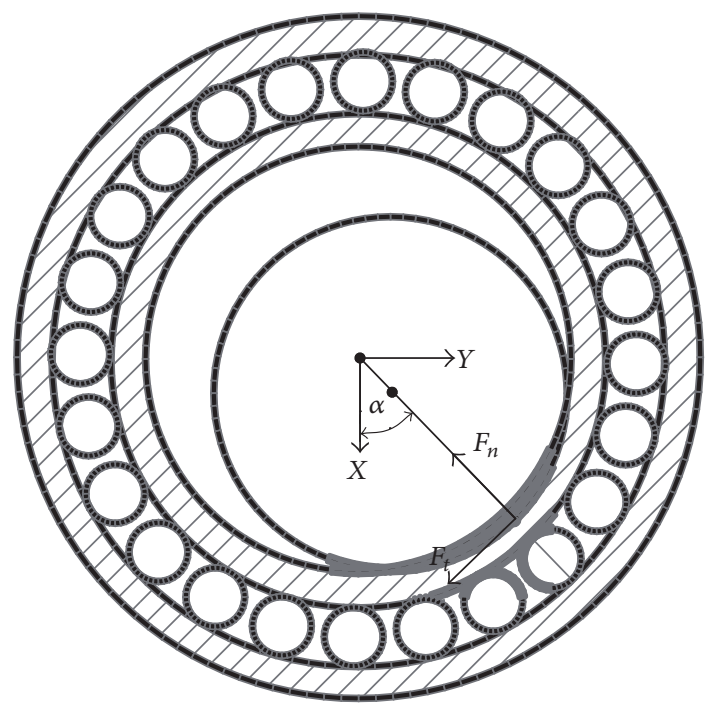

FIGURE 1: Sketch of auxiliary bearing and contact forces.

the auxiliary bearing is regarded as a whole, and the dynamic characteristics of auxiliary bearing parts and interactions between these parts are presented by the contact model with variable mechanical parameters, which will be discussed in this section.

On the other hand, as discussed in $[28,29]$ and this paper, the major impact forces occur at the first few impacts, this procedure usually takes only about one-tenth of a second, and the variation of the rotational speed of the rotor is neglectable. In this procedure, the motion state of the rotor is mainly affected by the impact forces, and the speeddependent forces, such as centrifugal and gyroscopic forces, play relatively minor roles. Therefore this paper concentrates on the impact forces between rotor and auxiliary bearing.

The rotor motion can be described by its displacements in three directions, namely, $x, y, z$, where $x$ and $y$ are the radial displacements and $z$ is the axial displacement. The inclination of the rotor is ignored in this paper.

3.2. Dynamic Model. Figure 1, which is a sketch of the auxiliary bearing, reveals contact forces. Throughout this paper, we assume that the rotor rotates in the anticlockwise direction.

The dynamic behavior of the rotor can be described by the following equations [13]:

$$
\begin{aligned}
& \ddot{x}=-\frac{F_{n}}{m} \cos \alpha+\frac{F_{t}}{m} \sin \alpha+e \omega^{2} \cos \theta, \\
& \ddot{y}=-\frac{F_{n}}{m} \sin \alpha-\frac{F_{t}}{m} \cos \alpha+e \omega^{2} \sin \theta, \\
& \ddot{z}=\frac{F_{z}}{m}-g, \\
& \theta=\theta_{0}+\omega t, \\
& \alpha=\arctan \frac{y}{x} .
\end{aligned}
$$

The definitions of notations are listed in the nomenclature. 
In the touchdown process, the dynamic behaviors of the rotor can be divided into a few classes according to the contact situations between the rotor and the auxiliary bearing. In the following part we divide the contact forces into two parts. The subscripts $R$ and $Z$ are utilized to denote the source of the contact force, namely, $F_{z}=F_{z R}+F_{z Z}$, for example. In this paper, the normal contact forces are described by the stiffness-damping model and the tangential contact forces by the friction model. Moreover, this paper assumes that the radial and the axial contact phenomena are decoupled. The detailed discussions of contact forces are given as follows:

(1) Radial contact

(a) No radial contact exists: the criterion of this case is $r \leq r_{0}$. The contact forces resulting by radial contact are all zero; namely,

$$
\begin{aligned}
& F_{n R}=0, \\
& F_{t R}=0, \\
& F_{z R}=0 .
\end{aligned}
$$

(b) Radial contact occurs: in this case, the radial force is given by the stiffness-damping model, and the tangent and axial forces are given by the friction model; namely,

$$
\begin{aligned}
& F_{n R}=k_{R}\left(r-r_{0}\right)+c_{R} \dot{r}, \\
& F_{t R}=\mu F_{n R}, \\
& F_{z R}=\mu \operatorname{sign}(\dot{z}) F_{n R} .
\end{aligned}
$$

(2) Axial contact

(a) No axial contact exists: the criterion of this case is $z \geq-z_{0}$. The contact forces resulting by axial contact are all zero; namely,

$$
\begin{aligned}
& F_{n Z}=0, \\
& F_{t Z}=0, \\
& F_{z Z}=0 .
\end{aligned}
$$

(b) Axial contact occurs: in this case, the axial force is obtained by the stiffness-damping model, and the radial force is zero. The tangent force, estimated by theoretical derivations and experiments, is proportional to the axial positive pressure due to friction. It is important to notice the positive pressure is exactly $F_{z Z}$. The force $F_{z R}$ resulted by the friction and will not lead to tangent force. From this discussion,

$$
\begin{aligned}
& F_{n Z}=0, \\
& F_{t Z}=k_{Z R} F_{z Z}, \\
& F_{z Z}=-k_{Z}\left(z+z_{0}\right)-c_{Z} \dot{z} .
\end{aligned}
$$

3.3. Learning-Based Model of Parameters. The model discussed in the above subsection involves some mechanical parameters, such as $k_{R}, c_{R}$. In [13], these parameters are regarded as constants and these values are determined theoretically or empirically. However, in this paper, these parameters are modeled as functions of auxiliary bearing deformation and rotor velocity. These function relationships can hardly be determined theoretically or experimentally. Thus this paper uses PKBKR technique to model these relationships. To be more specific, we use the PKBKR to model the following parameters:

$$
\begin{array}{r}
k_{R}(\boldsymbol{\rho})=\mathbf{Q}_{\boldsymbol{\rho}, T_{R}} \boldsymbol{\alpha}_{1}, \\
c_{R}(\boldsymbol{\rho})=\mathbf{Q}_{\boldsymbol{\rho}, T_{R}} \boldsymbol{\alpha}_{2}, \\
\mu(\boldsymbol{\rho})=\mathbf{Q}_{\boldsymbol{\rho}, T_{R}} \boldsymbol{\alpha}_{3}, \\
k_{Z}(\boldsymbol{\zeta})=\mathbf{Q}_{\zeta, T_{Z}} \boldsymbol{\alpha}_{4}, \\
c_{Z}(\boldsymbol{\zeta})=\mathbf{Q}_{\zeta, T_{Z}} \boldsymbol{\alpha}_{5}, \\
k_{Z R}(\boldsymbol{\zeta})=\mathbf{Q}_{\zeta, T_{Z}} \boldsymbol{\alpha}_{6},
\end{array}
$$

where

$$
\begin{aligned}
& \boldsymbol{\rho}=\left[\begin{array}{c}
r-r_{0} \\
\dot{r}
\end{array}\right], \\
& \boldsymbol{\zeta}=\left[\begin{array}{c}
z+z_{0} \\
\dot{z}
\end{array}\right]
\end{aligned}
$$

refer to the radial and axial deformation of auxiliary bearing and velocity of the rotor, respectively. Moreover, $T_{R}$ and $T_{Z}$ are predefined sample sets. The nominal values of these parameters are regarded as constant functions and utilized as the initial guesses of the models in (9a), (9b), (9c), (9d), (9e), and (9f). We formally denote these constant functions by $f_{0,1}, \ldots, f_{0,6}$, respectively. Their values can be found in [13]. The parameters $\boldsymbol{\alpha}_{1}, \ldots, \boldsymbol{\alpha}_{6}$ will be determined in the training process discussed in Section 4.3.

\section{State Estimation and Model Training}

Kalman filtering is an effective algorithm for state estimation based on system model and observations. Nonlinear extended Kalman filtering can be applied to deal with the nonlinear discrete-time system.

4.1. State Space Description of Touchdown Process. To apply Kalman filtering the state space description should be established firstly. Define the state variable as

$$
\boldsymbol{\xi}=\left[\begin{array}{lllllll}
x & y & z & \theta & \dot{x} & \dot{y} & \dot{z}
\end{array}\right]^{\top} .
$$


Then through the discussions in the next section, the derivative of $\xi$ is

$$
\begin{aligned}
\dot{\boldsymbol{\xi}} & =\boldsymbol{\varphi}(\boldsymbol{\xi}) \\
& =\left[\begin{array}{c}
\dot{x} \\
\dot{y} \\
\dot{z} \\
\omega \\
m^{-1}\left(-F_{n} \cos \alpha+F_{t} \sin \alpha\right)+e \omega^{2} \cos \theta \\
m^{-1}\left(-F_{n} \sin \alpha-F_{t} \cos \alpha\right)+e \omega^{2} \sin \theta \\
m^{-1} F_{z}-g
\end{array}\right],
\end{aligned}
$$

where $\varphi$ is the state transfer function. The observations are given by the displacement sensors. The observation variable is defined as $\boldsymbol{\eta}=\left[\begin{array}{lll}x & y & z\end{array}\right]^{\top}$.

The derivative matrix of $\varphi$ with respect to the state variable $\boldsymbol{\xi}$, denoted by $\boldsymbol{\Phi}$, is also necessary for applying Kalman filtering. This procedure is quite tedious and the computation result is quite complex. Hence we only give a few components of this matrix as examples, and the others can be computed in a similar way

$$
\begin{aligned}
& \boldsymbol{\Phi}_{(5,1)}=\frac{\partial \ddot{x}}{\partial x}=\frac{1}{m}\left(-\frac{\partial F_{n}}{\partial x} \cos \alpha-F_{n} \frac{\partial \cos \alpha}{\partial x}\right. \\
& \left.+\frac{\partial F_{t}}{\partial x} \sin \alpha+F_{t} \frac{\partial \sin \alpha}{\partial x}\right), \\
& \boldsymbol{\Phi}_{(5,5)}=\frac{\partial \ddot{x}}{\partial \dot{x}}=\frac{1}{m}\left(-\frac{\partial F_{n}}{\partial \dot{x}} \cos \alpha+\frac{\partial F_{t}}{\partial \dot{x}} \sin \alpha\right) \text {, } \\
& \frac{\partial F_{n R}}{\partial x} \\
& = \begin{cases}0, & r \leq r_{0}, \\
k_{R} \frac{\partial r}{\partial x}+\frac{\partial k_{R}}{\partial x}\left(r-r_{0}\right)+c_{R} \frac{\partial \dot{r}}{\partial x}+\frac{\partial c_{R}}{\partial x} \dot{r}, & r>r_{0},\end{cases} \\
& \frac{\partial F_{t R}}{\partial x}=\mu \frac{\partial F_{n R}}{\partial x}+F_{n R} \frac{\partial \mu}{\partial x}, \\
& \frac{\partial F_{n Z}}{\partial x}=\frac{\partial F_{t Z}}{\partial x}=0, \\
& \dot{r}=\frac{x}{r} \dot{x}+\frac{y}{r} \dot{y}=\cos \alpha \dot{x}+\sin \alpha \dot{y}, \\
& \frac{\partial r}{\partial x}=\frac{x}{r}=\cos \alpha, \\
& \frac{\partial \dot{r}}{\partial x}=\frac{y^{2}}{r^{3}} \dot{x}-\frac{x y}{r^{3}} \dot{y},
\end{aligned}
$$

$$
\begin{aligned}
& \frac{\partial \cos \alpha}{\partial x}=\frac{y^{2}}{r^{3}} \\
& \frac{\partial \sin \alpha}{\partial x}=\frac{-x y}{r^{3}} \\
& \frac{\partial F_{n R}}{\partial \dot{x}}= \begin{cases}0, & r \leq r_{0}, \\
\frac{\partial k_{R}}{\partial \dot{x}}\left(r-r_{0}\right)+c_{R} \frac{\partial \dot{r}}{\partial \dot{x}}+\dot{r} \frac{\partial c_{R}}{\partial \dot{x}}, & r>r_{0},\end{cases} \\
& \frac{\partial F_{t R}}{\partial \dot{x}}=\mu \frac{\partial F_{n R}}{\partial \dot{x}}+F_{n R} \frac{\partial \mu}{\partial \dot{x}}, \\
& \frac{\partial \dot{r}}{\partial \dot{x}}=\cos \alpha \\
& \frac{\partial k_{R}}{\partial x}=\left(\frac{\partial r}{\partial x}\left(\frac{\partial}{\partial r} \mathbf{Q}_{\boldsymbol{\rho}, T_{R}}\right)+\frac{\partial \dot{r}}{\partial x}\left(\frac{\partial}{\partial \dot{r}} \mathbf{Q}_{\boldsymbol{\rho}, T_{R}}\right)\right) \boldsymbol{\alpha}_{1}, \\
& \frac{\partial k_{R}}{\partial \dot{x}}=\frac{\partial \dot{r}}{\partial \dot{x}}\left(\frac{\partial}{\partial \dot{r}} \mathbf{Q}_{\boldsymbol{\rho}, T_{R}}\right) \boldsymbol{\alpha}_{1} \text {, } \\
& \frac{\partial c_{R}}{\partial x}=\left(\frac{\partial r}{\partial x}\left(\frac{\partial}{\partial r} \mathbf{Q}_{\boldsymbol{\rho}, T_{R}}\right)+\frac{\partial \dot{r}}{\partial x}\left(\frac{\partial}{\partial \dot{r}} \mathbf{Q}_{\boldsymbol{\rho}, T_{R}}\right)\right) \boldsymbol{\alpha}_{2}, \\
& \frac{\partial c_{R}}{\partial \dot{x}}=\frac{\partial \dot{r}}{\partial \dot{x}}\left(\frac{\partial}{\partial \dot{r}} \mathbf{Q}_{\boldsymbol{\rho}, T_{R}}\right) \boldsymbol{\alpha}_{2}, \ldots
\end{aligned}
$$

4.2. Nonlinear Extended Kalman Filtering. In the last subsection, the system is described by the following time-invariant continuous-time state space description:

$$
\begin{aligned}
& \dot{\xi}=\varphi(\xi), \\
& \eta=\mathbf{H} \xi
\end{aligned}
$$

where $\boldsymbol{\xi}$ and $\boldsymbol{\eta}$ are the state variable and the observation variable, $\boldsymbol{\varphi}$ is the state transfer function, and $\mathbf{H}$ is the observation matrix. In order to discretize the model (14a) and (14b) to a discrete-time model, the Runge-Kutta [30] method is utilized, and then the discrete-time model is in the following iterative form:

$$
\begin{aligned}
\boldsymbol{\xi}_{k+1} & =\mathbf{f}\left(\boldsymbol{\xi}_{k}\right)=\boldsymbol{\xi}_{k}+\frac{1}{6}\left(\mathbf{k}_{1}+2 \mathbf{k}_{2}+2 \mathbf{k}_{3}+\mathbf{k}_{4}\right), \\
\mathbf{k}_{1} & =h \boldsymbol{\varphi}\left(\boldsymbol{\xi}_{k}\right) \\
\mathbf{k}_{2} & =h \varphi\left(\boldsymbol{\xi}_{k}+\frac{1}{2} \mathbf{k}_{1}\right), \\
\mathbf{k}_{3} & =h \boldsymbol{\varphi}\left(\boldsymbol{\xi}_{k}+\frac{1}{2} \mathbf{k}_{2}\right), \\
\mathbf{k}_{4} & =h \boldsymbol{\varphi}\left(\boldsymbol{\xi}_{k}+\mathbf{k}_{3}\right), \\
\boldsymbol{\eta}_{k} & =\mathbf{H} \boldsymbol{\xi}_{k},
\end{aligned}
$$

where $h$ is the sampling period.

The first-order derivative matrix of $\mathbf{f}$ should be evaluated to apply Kalman filtering. Through tedious computations, the 
derivative matrix $\boldsymbol{\Phi}_{k}=\left.(\partial \mathbf{f} / \partial \mathbf{z})\right|_{\left(\xi_{k}\right)}$ can be written in the iterative form [23]:

$$
\begin{aligned}
& \Phi_{k}=\mathbf{I}+\left.\frac{1}{6}\left(\frac{\partial \mathbf{k}_{1}}{\partial \xi}+2 \frac{\partial \mathbf{k}_{2}}{\partial \mathbf{z}}+2 \frac{\partial \mathbf{k}_{3}}{\partial \xi}+\frac{\partial \mathbf{k}_{4}}{\partial \xi}\right)\right|_{\left(\xi_{k}\right)}, \\
& \left.\frac{\partial \mathbf{k}_{1}}{\partial \xi}\right|_{\left(\xi_{k}\right)}=\left.h \frac{\partial \varphi}{\partial \xi}\right|_{\left(\xi_{k}\right)} \text {, } \\
& \left.\frac{\partial \mathbf{k}_{2}}{\partial \boldsymbol{\xi}}\right|_{\left(\xi_{k}\right)}=\left.h \frac{\partial \boldsymbol{\varphi}}{\partial \boldsymbol{\xi}}\right|_{\left(\xi_{k}+(1 / 2) \mathbf{k}_{1}\right)}\left(\mathbf{I}+\left.\frac{1}{2} \frac{\partial \mathbf{k}_{1}}{\partial \boldsymbol{\xi}}\right|_{\left(\xi_{k}\right)}\right) \text {, } \\
& \left.\frac{\partial \mathbf{k}_{3}}{\partial \xi}\right|_{\left(\xi_{k}\right)}=\left.h \frac{\partial \varphi}{\partial \xi}\right|_{\left(\xi_{k}+(1 / 2) \mathbf{k}_{2}\right)}\left(\mathbf{I}+\left.\frac{1}{2} \frac{\partial \mathbf{k}_{2}}{\partial \xi}\right|_{\left(\xi_{k}\right)}\right) \text {, } \\
& \left.\frac{\partial \mathbf{k}_{4}}{\partial \boldsymbol{\xi}}\right|_{\left(\xi_{k}\right)}=\left.h \frac{\partial \boldsymbol{\varphi}}{\partial \boldsymbol{\xi}}\right|_{\left(\xi_{k}+\mathbf{k}_{3}\right)}\left(\mathbf{I}+\left.\frac{\partial \mathbf{k}_{3}}{\partial \boldsymbol{\xi}}\right|_{\left(\xi_{k}\right)}\right) \text {, }
\end{aligned}
$$

where $\mathbf{I}$ is the identity matrix.

Then extended Kalman filtering can be applied to compute the rotor displacement estimation $\widetilde{\xi}_{k}$. The detailed estimation program is standard, and we write down it here for completeness

$$
\begin{aligned}
\widetilde{\boldsymbol{\xi}}_{k,-} & =\mathbf{f}\left(\widetilde{\boldsymbol{\xi}}_{k-1}\right), \\
\widetilde{\boldsymbol{\eta}}_{k} & =\mathbf{H} \widetilde{\boldsymbol{\xi}}_{k,-}, \\
\widetilde{\boldsymbol{\xi}}_{k} & =\widetilde{\boldsymbol{\xi}}_{k,-}+\mathbf{K}_{k}\left(\boldsymbol{\eta}_{k}-\widetilde{\boldsymbol{\eta}}_{k}\right), \\
\mathbf{P}_{k,-} & =\boldsymbol{\Phi}_{k} \mathbf{P}_{k-1} \boldsymbol{\Phi}_{k}^{\top}+\mathbf{Q}, \\
\mathbf{K}_{k} & =\mathbf{P}_{k,-}\left(\mathbf{H} \mathbf{P}_{k,-} \mathbf{H}^{\top}+\mathbf{R}\right)^{-1}, \\
\mathbf{P}_{k} & =\left(\mathbf{I}-\mathbf{K}_{k} \mathbf{H}\right) \mathbf{P}_{k,-},
\end{aligned}
$$

where $\widetilde{\boldsymbol{\xi}}_{0}, \mathbf{P}_{0}, \mathbf{Q}, \mathbf{R}$ are predefined parameters.

4.3. Model Training. Suppose that all mechanical parameters are given and a measured data set $\left\{\boldsymbol{\eta}_{k}\right\}_{k=1}^{l}$ is available, given the coefficients $\boldsymbol{\alpha}_{1}, \ldots, \boldsymbol{\alpha}_{6}$, and then the system state and output $\left\{\widetilde{\boldsymbol{\xi}}, \widetilde{\boldsymbol{\eta}}_{k}\right\}_{k=1}^{l}$ can be estimated based on the dynamic model and the state estimation technique discussed in the above subsections. Therefore the estimated output $\left\{\widetilde{\boldsymbol{\eta}}_{k}\right\}_{k=1}^{l}$ depends on the coefficients $\boldsymbol{\alpha}_{1}, \ldots, \boldsymbol{\alpha}_{6}$ and can be utilized to evaluate the precision of PKBKR model. More specifically, we use the following minimization problem to train these parameters:

$$
\begin{aligned}
\min _{\boldsymbol{\alpha}_{1}, \ldots, \boldsymbol{\alpha}_{6}} & \sum_{k=1}^{l}\left\|\tilde{\boldsymbol{\eta}}_{k}-\boldsymbol{\eta}_{k}\right\|^{2} \\
& +\lambda\left(\sum_{k=1}^{3}\left\|\mathbf{Q}_{\boldsymbol{\rho}, T_{R}} \boldsymbol{\alpha}_{k}-f_{0, k}\right\|_{\mathscr{H}}^{2}+\sum_{k=4}^{6}\left\|\mathbf{Q}_{\zeta, T_{Z}} \boldsymbol{\alpha}_{k}-f_{0, k}\right\|_{\mathscr{H}}^{2}\right),
\end{aligned}
$$

where $\lambda$ is the regularization parameter and $\mathscr{H}$ is the reproducing kernel Hilbert space $[31,32]$ associated with the kernel function $Q$. The regularization term $\lambda\left(\sum \| \mathbf{Q}_{\boldsymbol{\rho}, T_{R}} \boldsymbol{\alpha}_{k}-\right.$ $\left.f_{0, k}\left\|_{\mathscr{H}}^{2}+\sum\right\| \mathbf{Q}_{\zeta, T_{Z}} \boldsymbol{\alpha}_{k}-f_{0, k} \|_{\mathscr{H}}^{2}\right)$ represents the distance between
PKBKR models and initial guesses. The prior knowledge carried by the initial guesses is incorporated by introducing this term.

\section{Experiments}

5.1. Experiment Setup. Experiments are performed to validate the proposed method. The data in the experiments are attained from 21 touchdown experiments on the backup helium circulator system of HTR-10. A detailed description of this system can be found in $[11,13,33]$. These experiments are performed with various conditions; for example, the brake of drive motor will be applied and an additional axial load (about $2000 \mathrm{~N}$ ) will be applied by an axial loader [29]. The experiment conditions are summarized in Table 1.

In the analysis, the data in the first 0.15 seconds after the touchdown is used. The proposed method is utilized to estimate the state of the system. Moreover, the results in [13], which are produced by a method without machine learning modeling, are also illustrated for comparison.

5.2. Results of Displacement Estimation. Firstly, the results of rotor displacement estimation in experiment \#11 are shown in Figures 2-4. In these figures, the measured displacements are denoted by $x, y, z$. Notations $x_{\text {est }}, y_{\text {est }}, z_{\text {est }}$ refer to the displacements estimated by the method in [13] and $x_{\text {est,PKBKR }}, y_{\text {est,PKBKR }}, z_{\text {est,PKBKR }}$ stand for estimation results of the proposed method.

Moreover, the displacement estimation errors of all 21 experiments are shown in Figure 5, where the estimation error is evaluated by the root mean square error as

$$
x_{\mathrm{err}}=\sqrt{\frac{1}{l} \sum_{k=1}^{l}\left(x_{\mathrm{est}, k}-x_{k}\right)^{2}},
$$

$$
x_{\text {err,PKBKR }}=\sqrt{\frac{1}{l} \sum_{k=1}^{l}\left(x_{\text {est,PKBKR }, k}-x_{k}\right)^{2}} \text {, }
$$

$$
y_{\mathrm{err}}=\sqrt{\frac{1}{l} \sum_{k=1}^{l}\left(y_{\mathrm{est}, k}-y_{k}\right)^{2}} \text {, }
$$

$$
y_{\text {err,PKBKR }}=\sqrt{\frac{1}{l} \sum_{k=1}^{l}\left(y_{\text {est,PKBKR }, k}-y_{k}\right)^{2}} \text {, }
$$

$$
z_{\text {err }}=\sqrt{\frac{1}{l} \sum_{k=1}^{l}\left(z_{\text {est }, k}-z_{k}\right)^{2}}
$$

$$
z_{\text {err,PKBKR }}=\sqrt{\frac{1}{l} \sum_{k=1}^{l}\left(z_{\mathrm{est}, \mathrm{PKBKR}, k}-z_{k}\right)^{2}},
$$

where $l$ denotes the size of data set, $x_{k}, x_{\text {est }, k}, x_{\text {est,PKBKR, } k}$ denote $k$-th sample of $x, x_{\text {est }}, x_{\text {est,PKBKR, },}$, respectively, and other notations are self-explanatory. 
TABLE 1: Summation of touchdown experiments.

\begin{tabular}{|c|c|c|c|c|}
\hline Number of experiments & Touchdown speed (rpm) & Brake? & Additional axial load? & Auxiliary bearing type \\
\hline 1 & 5000 & No & No & Ceramic ball \\
\hline 2 & 5000 & No & No & Ceramic ball \\
\hline 3 & 5000 & No & No & Ceramic ball \\
\hline 4 & 5000 & No & No & Ceramic ball \\
\hline 5 & 5000 & No & No & Ceramic ball \\
\hline 6 & 5000 & Yes & No & Ceramic ball \\
\hline 7 & 5000 & Yes & No & Ceramic ball \\
\hline 8 & 5000 & Yes & Yes & Ceramic ball \\
\hline 9 & 5000 & Yes & Yes & Ceramic ball \\
\hline 10 & 5000 & Yes & Yes & Ceramic ball \\
\hline 11 & 5000 & Yes & Yes & Ceramic ball \\
\hline 12 & 5000 & Yes & Yes & Ceramic ball \\
\hline 13 & 5000 & Yes & No & Ceramic ball \\
\hline 14 & 5000 & Yes & No & Ceramic ball \\
\hline 15 & 5000 & Yes & Yes & Ceramic ball \\
\hline 16 & 5000 & Yes & Yes & Ceramic ball \\
\hline 17 & 5000 & Yes & Yes & Ceramic ball \\
\hline 18 & 5000 & Yes & Yes & Ceramic ball \\
\hline 19 & 5000 & Yes & Yes & Ceramic ball \\
\hline 20 & 5000 & Yes & Yes & Ceramic ball \\
\hline 21 & 5000 & No & No & Steel ball \\
\hline
\end{tabular}

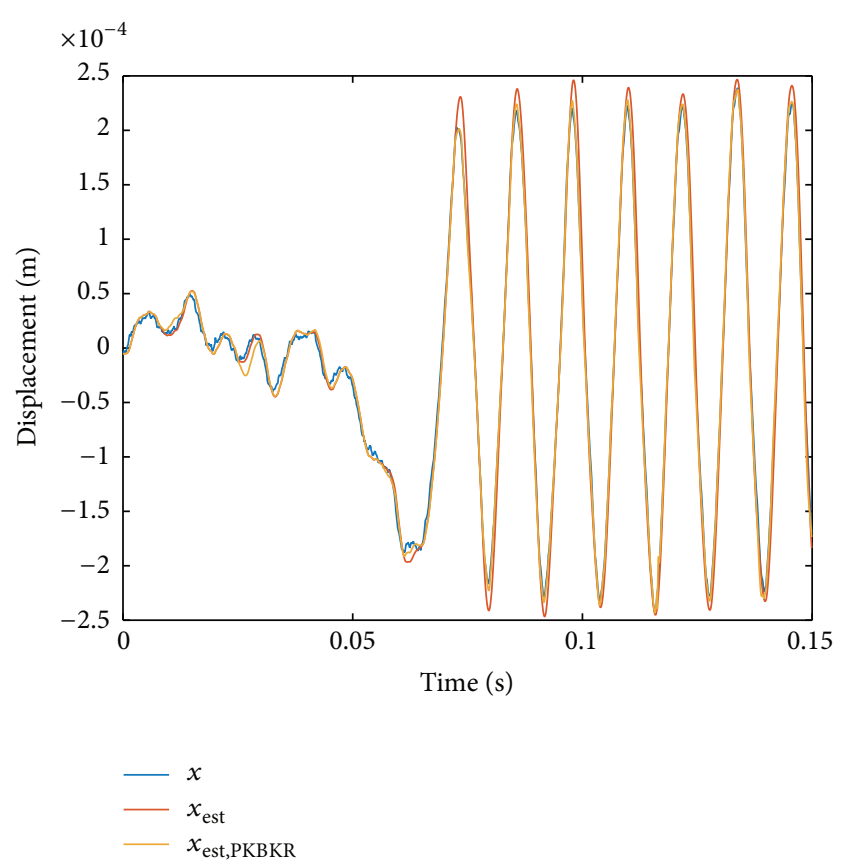

Figure 2: Measured and estimated displacements. $X$-direction, experiment \#11.

As shown in Figure 5, precise estimations of the displacement of the rotor can be achieved by the proposed method under various conditions in all 21 experiments, and the root mean square errors in all three directions are less

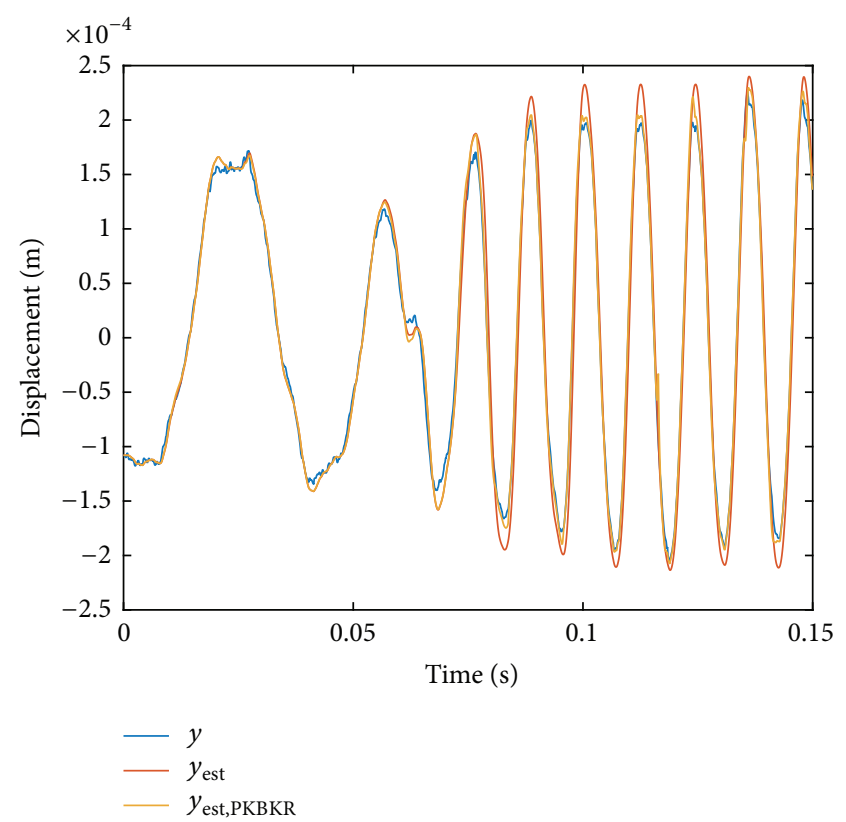

FIGURE 3: Measured and estimated displacements. Y-direction, experiment \#11.

than $0.02 \mathrm{~mm}$. Thus the proposed method is suitable for analyzing the touchdown process under various conditions.

It is obvious that the proposed method makes an excellent estimation of rotor displacement and outperforms the 


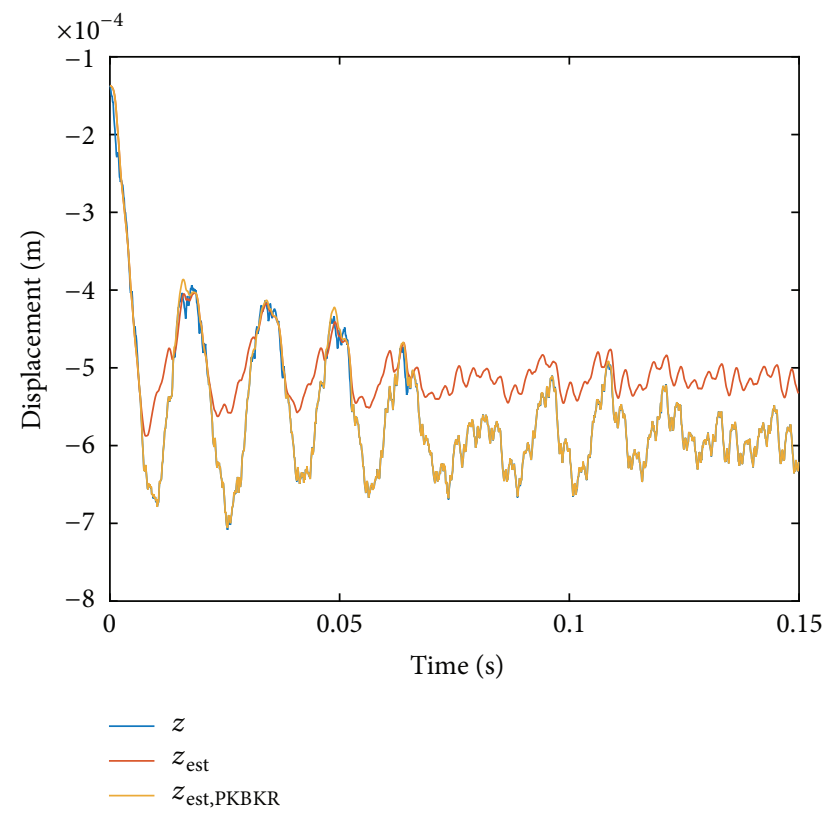

FIGURE 4: Measured and estimated displacements. Z-direction, experiment \#11.
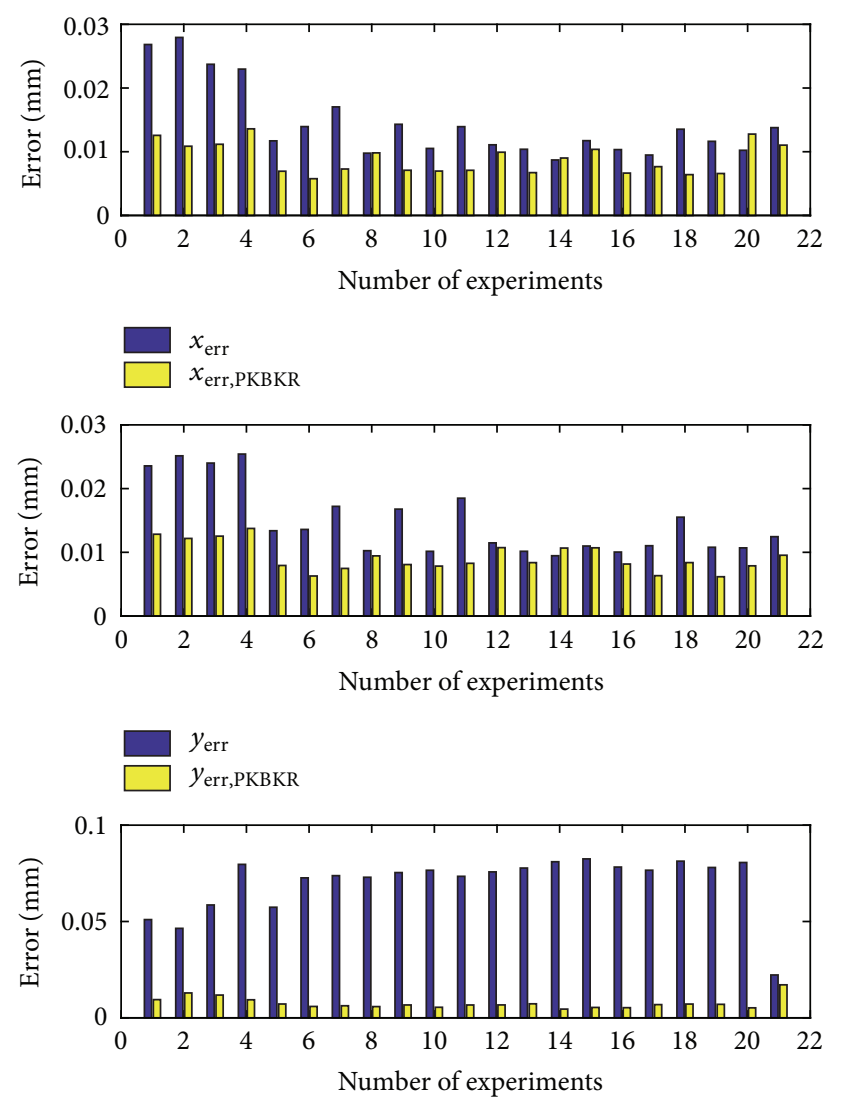

$z_{\text {err }}$

$z_{\text {err,PKBKR }}$

FIgURE 5: Displacement estimation errors. All directions and all 21 experiments.

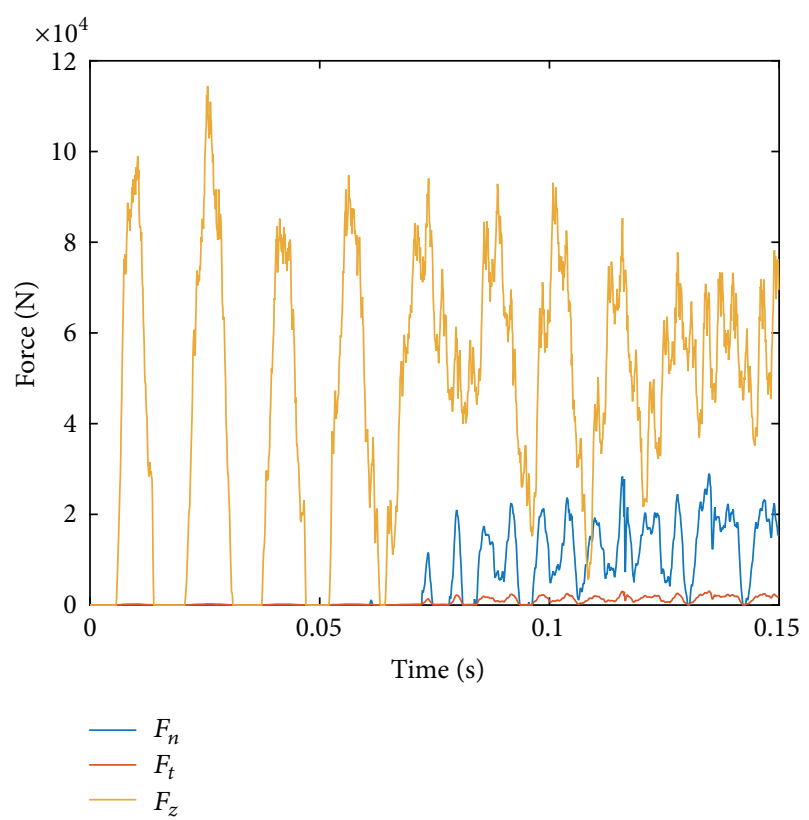

FIGURE 6: Estimated force. Experiment \#11.

method without learning, especially in the estimation of $z$ displacement.

The main reason is that, in the original dynamic model, some mechanical parameters are assumed to be constant, but in practice these parameters may be affected by the motion of rotor and auxiliary bearing. By introducing learning method, these parameters are allowed to vary with respect to rotor motion and the quality of the dynamic model is significantly improved. Moreover, the relationship between these parameters and the rotor motion is determined by the measured data; thus precise estimations are achieved. On the other hand, as for the precision of radial displacement estimation, both methods (with and without learning) are similar, shown in Figure 5. The main reason is that, compared with complicated axial motion, the radial motion of the rotor is relatively simpler and smoother. As shown in Figure 5, the rotor carries out circular motion after first few impacts and radial displacements in $X$ - and $Y$-direction are sinusoidal. The radial acceleration, velocity, and deformation of the auxiliary bearing are relatively smaller; thus the radial mechanical parameters do not vary significantly and the estimation method with constant methods also achieves satisfactory performance.

5.3. Results of Force Estimation. The estimated contact force in experiment \#11 is shown in Figure 6. The maximal impact occurs at the first touchdown. The touchdown process can be divided into two stages: in the first six bounces, no radial contact between the rotor and auxiliary bearing occurs and the radial forces can be neglected; after the sixth bounces, the rotor clings to the auxiliary bearing and large radial forces emerge. The radial force is dominated by the normal force. 


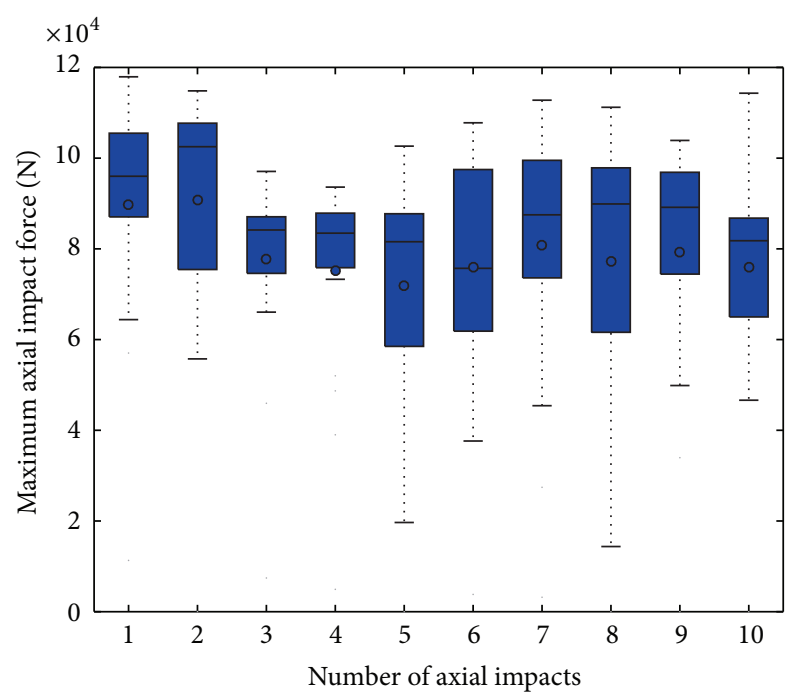

Figure 7: Estimated axial force. All 21 experiments.

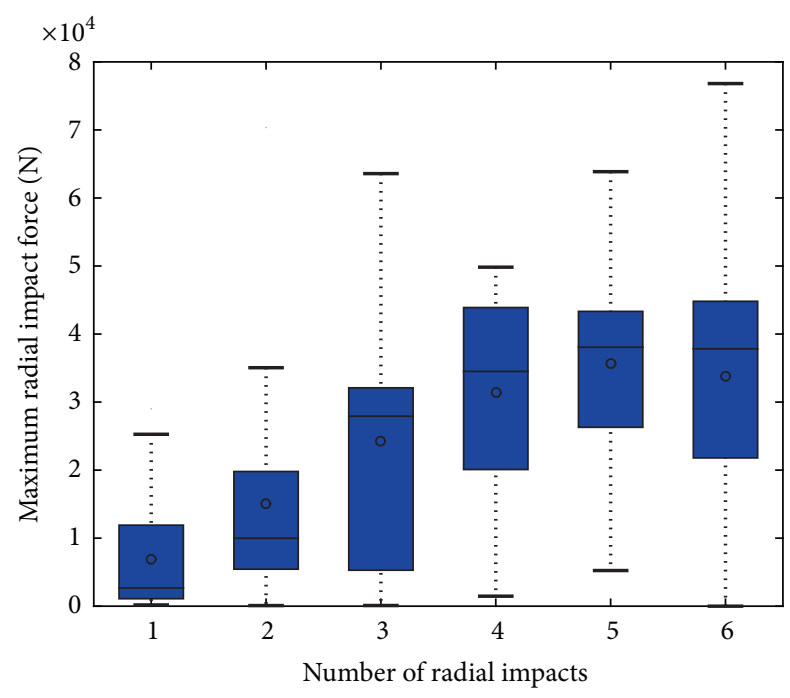

Figure 8: Estimated normal force. All 21 experiments.

The experiment results have shown the validity of the proposed method in estimating the motion state of the rotor during the touchdown process.

Furthermore, in order to visualize the force estimations in all 21 experiments, the peak values of axial and radial forces are extracted. The peak values are grouped according to the number of impacts. The statistical distributions are illustrated in the form of boxplot, shown in Figures 7 and 8. From these figures, a similar pattern can be found; namely, the maximal axial impact occurs at the first two touchdowns and the peak values of axial force decrease slowly in the following touchdowns and bounces. As above mentioned, the radial forces emerge after the first radial contact between rotor and bearing, and the peak values of radial force increase at the first several radial impacts. The maximal radial force is about one-third to one-half of the maximal axial force.

\section{Conclusions and Discussions}

Magnetic bearings are widely applied in High Temperature Gas-cooled Reactor (HTGR) and auxiliary bearings are important backup and safety components in AMB systems. In the applications associated with HTGR, the performance of auxiliary bearings significantly affects the reliability, safety, and serviceability of the AMB system, the rotating equipment, and the whole reactor. The dynamic model of the touchdown process is firstly established. In this model, some mechanical parameters are regarded as functions of deformation of auxiliary bearing and velocity of the rotor and a machine learning technique is utilized to model these function relationships. Based on the dynamic model and Kalman filtering technique, the proposed method can offer estimations of rotor's motion state from noisy observations and estimation precision is significantly improved compared with the method without learning. The proposed method is validated by the experimental data from touchdown experiments.

The proposed method in this paper provides a novel data processing method for the touchdown process and is a foundation for further researches.

The future work includes the following:

(1) As above mentioned, this paper depends on the actual touchdown data, which cannot be directly utilized in the design stage of the whole rotating equipment. In order to evaluate the severity of touchdown and improve the auxiliary bearing design, analysis of strain and stress in the auxiliary bearing will be performed based on the estimated force. Moreover, the operational state and remaining life of auxiliary bearings can be estimated as well, as discussed in $[7,24]$. Together with these works, the design-verification-validation loop can be completed.

(2) In this paper, a relatively simple motion model is applied. Only one axial-radial auxiliary bearing and three degree-of-freedom of the rotor are considered, the eccentricity is constant, and the gyroscopic force is ignored. In the future work, we intend to apply the proposed method to the five degree-of-freedom rotor-bearing model [28]. Furthermore, the overall motion model considering the interaction between the rotor, inner ring, balls, and outer ring of the auxiliary bearing will be analyzed in the future.

(3) In this paper, 21 experiments are analyzed and conclusions are plotted in a statistical sense. In the future, more touchdown experiments are planned to make more precise statistical analyses of the performance of auxiliary bearings, touchdown conditions, and their effect on the whole reactor under accident conditions.

\section{Nomenclature}

$\alpha: \quad$ Contact angle between the rotor and the auxiliary bearing

$\ddot{x}, \ddot{y}, \ddot{z}$ : Second-order derivatives of displacements, namely, the accelerations of the rotor

$\dot{x}, \dot{y}, \dot{z}$ : Derivatives of displacements, namely, the velocities of the rotor 


\begin{tabular}{|c|c|}
\hline$\rho:$ & $\begin{array}{l}\text { Vector of auxiliary bearing radial } \\
\text { deformation and rotor radial velocity }\end{array}$ \\
\hline$\zeta$ & $\begin{array}{l}\text { Vector of auxiliary bearing axial } \\
\text { deformation and rotor axial velocity }\end{array}$ \\
\hline$\mu:$ & $\begin{array}{l}\text { Friction coefficient between the rotor and } \\
\text { the auxiliary bearing }\end{array}$ \\
\hline$\omega:$ & Rotational speed of the rotor \\
\hline sign: & Signum function \\
\hline$\theta:$ & Rotational angle of the rotor \\
\hline$\theta_{0}:$ & $\begin{array}{l}\text { Rotational angle of the rotor at the instant } \\
\text { of the shutdown of magnetic bearings }\end{array}$ \\
\hline$c_{R}, c_{Z}:$ & Radial and axial damping coefficient \\
\hline$e:$ & $\begin{array}{l}\text { Eccentricity between the mass center and } \\
\text { the rotational center of the rotor }\end{array}$ \\
\hline$F_{n}, F_{t}, F_{z}:$ & $\begin{array}{l}\text { Radial, tangential, and axial contact forces } \\
\text { between the rotor and the auxiliary } \\
\text { bearing }\end{array}$ \\
\hline$F_{n R}, F_{t R}, F_{z R}:$ & Contact forces caused by radial contact \\
\hline$F_{n Z}, F_{t Z}, F_{z Z}$ & Contact forces caused by axial contact \\
\hline$g:$ & Acceleration due to gravity \\
\hline$k_{R}, k_{Z}:$ & Radial and axial stiffness \\
\hline$k_{Z R}:$ & $\begin{array}{l}\text { Coefficient between tangential force and } \\
\text { axial positive pressure }\end{array}$ \\
\hline$m:$ & Mass of the rotor \\
\hline Q: & Kernel function \\
\hline$r:$ & Radial displacement of the rotor \\
\hline$r_{0}, z_{0}:$ & $\begin{array}{l}\text { Nominal radial and axial clearance } \\
\text { between the rotor and the auxiliary } \\
\text { bearing at operation position }\end{array}$ \\
\hline$t:$ & $\begin{array}{l}\text { Time elapsed since the shutdown of } \\
\text { magnetic bearings }\end{array}$ \\
\hline$T_{R}, T_{Z}$ & Sample sets \\
\hline$x, y, z:$ & $\begin{array}{l}\text { Displacements of the rotor in three } \\
\text { directions }\end{array}$ \\
\hline$\alpha_{1}, \ldots, \alpha_{6}:$ & Coefficients in learning models. \\
\hline
\end{tabular}

\section{Conflicts of Interest}

The authors declare that there are no conflicts of interest regarding the publication of this paper.

\section{Acknowledgments}

This paper is financially supported by the National Science and Technology Major Project of China (2011ZX069) and Project 61305065 and Project 51275261 supported by NSFC.

\section{References}

[1] T. Ishii and R. G. Kirk, "Transient response technique applied to active magnetic bearing machinery during rotor drop," Journal of Rotating Machinery and Vehicle Dynamics, pp. 191-199, 1991.

[2] M. Fumagalli and G. Schweitzer, "Measurements on a rotor contacting its housing," in Proceedings of the in Imeche Conference Transactions, vol. 6, pp. 779-788, 1996.

[3] Z. Fang and L. E. Barrett, "Transient response of single disk rotor with active magnetic bearings during rotor drop," Journal of Shanghai Jiaotong University, p. 7, 1996.
[4] S. Zeng, "Modelling and experimental study of the transient response of an active magnetic bearing rotor during rotor drop on back-up bearings," Proceedings of the Institution of Mechanical Engineers, Part I: Journal of Systems and Control Engineering, vol. 217, no. 6, pp. 505-517, 2003.

[5] G. Sun, A. B. Palazzolo, A. Provenza, and G. Montague, "Detailed ball bearing model for magnetic suspension auxiliary service," Journal of Sound and Vibration, vol. 269, no. 3-5, pp. 933-963, 2004.

[6] G. Sun, "Rotor drop and following thermal growth simulations using detailed auxiliary bearing and damper models," Journal of Sound and Vibration, vol. 289, no. 1-2, pp. 334-359, 2006.

[7] J. G. Lee and A. Palazzolo, "Catcher bearing life prediction using a rainflow counting approach," Journal of Tribology, vol. 134, no. 3, Article ID 031101, 2012.

[8] P. S. Keogh and W. Y. Yong, “Thermal assessment of dynamic rotor/auxiliary bearing contact events," Journal of Tribology, vol. 129, no. 1, pp. 143-152, 2007.

[9] M. Schlotter and P. S. Keogh, "Synchronous position recovery control for flexible rotors in contact with auxiliary bearings," Journal of Vibration and Acoustics, vol. 129, no. 5, pp. 550-558, 2007.

[10] Z.-W. Tan, L. Wan, and S.-Y. He, "Impact simulation of HTR10 vertical magnetic suspension rotor dropping into catcher bearings in early design phase," Hedongli Gongcheng/Nuclear Power Engineering, vol. 29, no. 5, pp. 5-8, 2008.

[11] J.-X. Zhao, G.-J. Yang, Y. Li, and S.-Y. Yu, "Numerical analysis of magnetically suspended rotor in htr-10 helium circulator being dropped into auxiliary bearings," Nuclear Power Engineering, vol. 33, no. 3, pp. 61-64, 2012.

[12] Z. Xiao, G. Yang, Z. Shi, and S. Yu, "Behavior of a magnetically suspended rotor dropped into auxiliary bearings," in Proceedings of the 2013 21st International Conference on Nuclear Engineering, ICONE 2013, August 2013.

[13] Z. Sun, X. Kang, J. Zhao, G. Yang, and Z. Shi, "Dynamic behavior analysis of touchdown process in active magnetic bearing system based on Kalman filtering," in Proceedings of the 2014 22nd International Conference on Nuclear Engineering, ICONE 2014, July 2014.

[14] W. Yan, H. Shao, and X. Wang, "Soft sensing modeling based on support vector machine and Bayesian model selection," Computers \& Chemical Engineering, vol. 28, no. 8, pp. 1489-1498, 2004.

[15] J. Luo and H. Shao, "Soft sensing modeling using neurofuzzy system based on rough set theory," in Proceedings of the 2002 American Control Conference, pp. 543-548, May 2002.

[16] S. C. Patwardhan, J. Prakash, and S. L. Shah, "Soft sensing and state estimation: review and recent trends," IFAC Proceedings Volumes, vol. 40, no. 19, pp. 65-72, 2007.

[17] K. Worden, W. J. Staszewski, and J. J. Hensman, "Natural computing for mechanical systems research: A tutorial overview," Mechanical Systems and Signal Processing, vol. 25, no. 1, pp. 4111, 2011.

[18] F. Wu and T. Chai, "Soft sensing method for magnetic tube recovery ratio via fuzzy systems and neural networks," Neurocomputing, vol. 73, no. 13-15, pp. 2489-2497, 2010.

[19] E. Terzic, R. Nagarajah, and M. Alamgir, "A neural network approach to fluid quantity measurement in dynamic environments," Mechatronics, vol. 21, no. 1, pp. 145-155, 2011.

[20] Y. Çaya, A. Çiçekb, F. Karac, and S. Sağiroğlua, "Prediction of engine performance for an alternative fuel using artificial 
neural network," Applied Thermal Engineering, vol. 37, pp. 217225, 2012.

[21] E. Kilic, C. U. Dogruer, M. Dolen, and A. B. Koku, "Position estimation for timing belt drives of precision machinery using structured neural networks," Mechanical Systems and Signal Processing, vol. 29, pp. 343-361, 2012.

[22] Z. Sun, J. Zhao, and S. Yu, "Nonlinear nonparametric identification of magnetic bearing system based on support vector regression," in Proceedings of the 13th International Symposium on Magnetic Bearings, 2012.

[23] Z. Sun, J. Zhao, Z. Shi, and S. Yu, "Soft sensing of magnetic bearing system based on support vector regression and extended Kalman filter," Mechatronics, vol. 24, no. 3, pp. 186-197, 2014.

[24] G. Sun, "Auxiliary bearing life prediction using Hertzian contact bearing model," Journal of Vibration and Acoustics, vol. 128, no. 2, pp. 203-209, 2006.

[25] Z. Sun, Z.-K. Zhang, and H.-G. Wang, "Incorporating prior knowledge into kernel based regression," Acta Automatica Sinica, vol. 34, no. 12, pp. 1515-1521, 2008.

[26] A. J. Smola and B. Schölkopf, "A tutorial on support vector regression," Statistics and Computing, vol. 14, no. 3, pp. 199-222, 2004.

[27] N. Cristianini and J. Shawe-Taylor, An Introduction to Support Vector Machines, Cambridge University Press, Cambridge,UK, 2000.

[28] Y. Zhao, G. Yang, X. Liu, Z. Shi, and L. Zhao, "Research on dynamics and experiments about auxiliary bearings for the helium circulator of the $10 \mathrm{MW}$ high temperature gas-cooled reactor," Annals of Nuclear Energy, vol. 95, pp. 176-187, 2016.

[29] Y. Zhao, G. Yang, P. Keogh, and L. Zhao, "Dynamic Analysis for the Rotor Drop Process and Its Application to a Vertically Levitated Rotor/Active Magnetic Bearing System," Journal of Tribology, vol. 139, no. 4, p. 041701, 2017.

[30] F. E. Cellier and E. Kofman, Continuous System Simulation, Springer Science+Business Media, Boston, Mass, USA, 2006.

[31] H. Meschkowski, Hilbertsche Räume mit Kernfunction, Die Grundlehren der mathematischen Wissenschaften, Bd. 113, Springer, Berlin, Germany, 1962.

[32] B. Schölkopf and A. J. Smola, Learning with Kernels: Support Vector Machines, Regularization, Optimization, and beyond, The MIT Press, Cambridge, Mass, USA, 2003.

[33] X. Kang, G. Yang, and S. Yu, "Dynamic behavior of the AMB's vertical arranged rotor during its drop process," in Proceedings of the 2014 22nd International Conference on Nuclear Engineering, ICONE 2014, July 2014. 


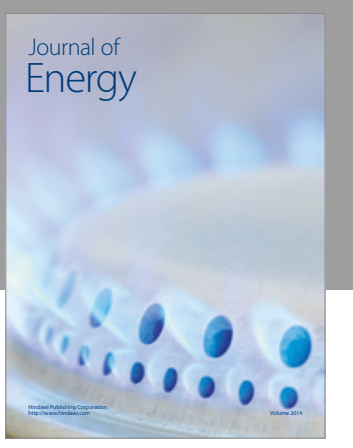

Journal of

Industrial Engineering
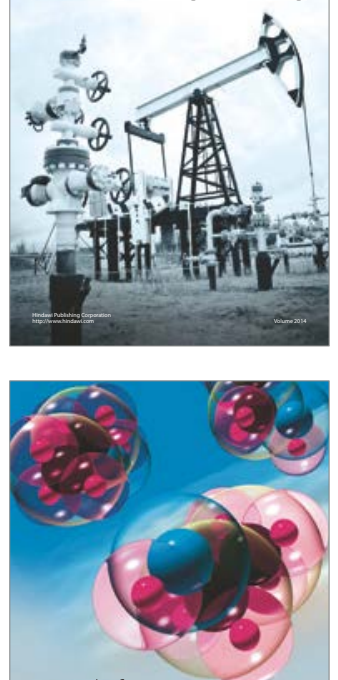

Fuels
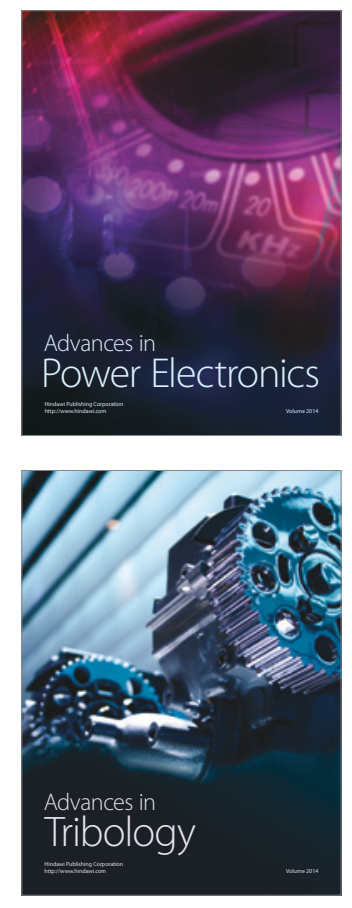
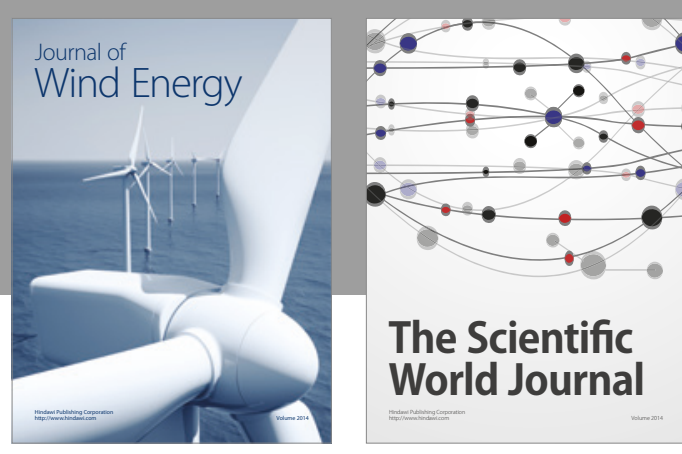

The Scientific World Journal
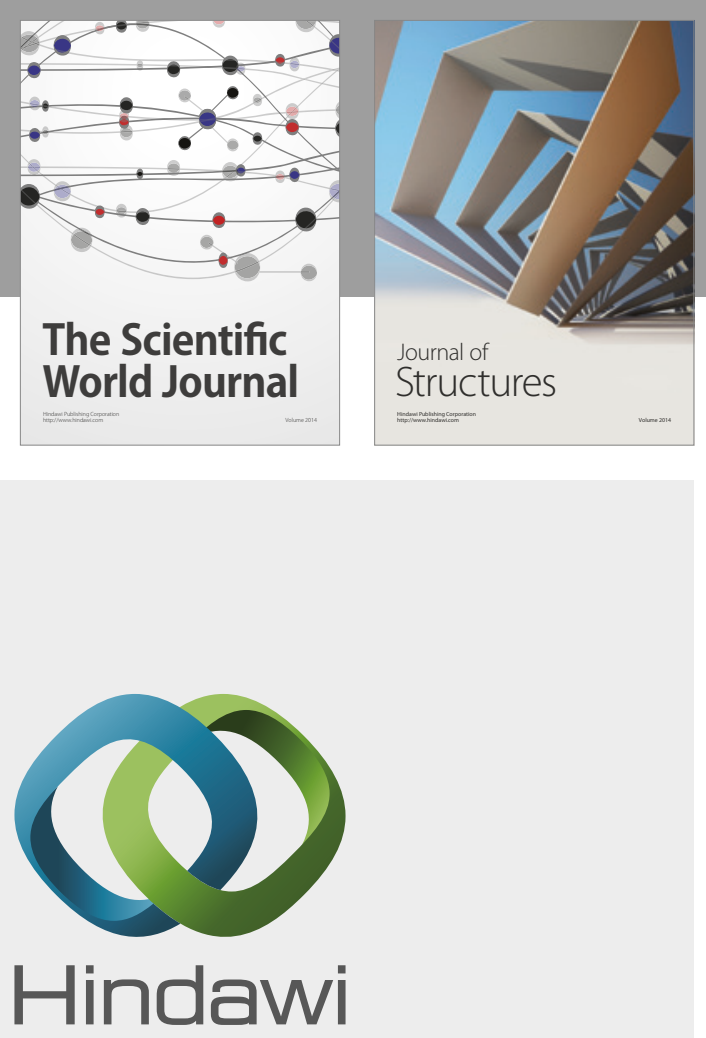

Submit your manuscripts at

https://www.hindawi.com
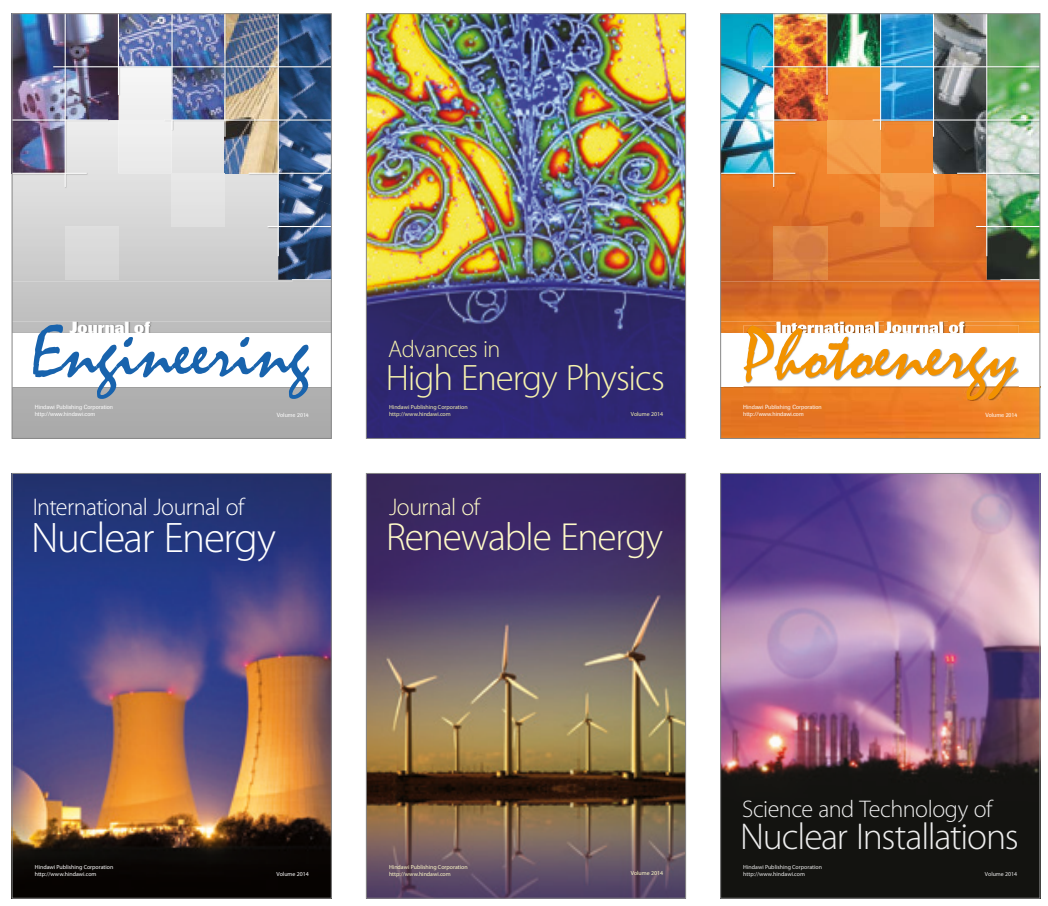

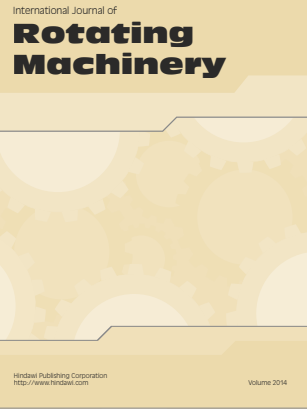

Journal of

Petroleum Engineering

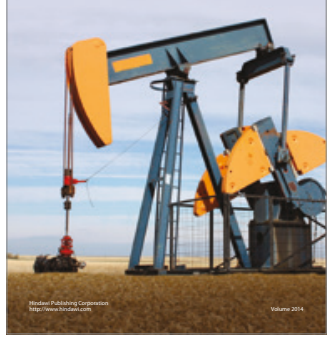

Journal of
Solar Energy
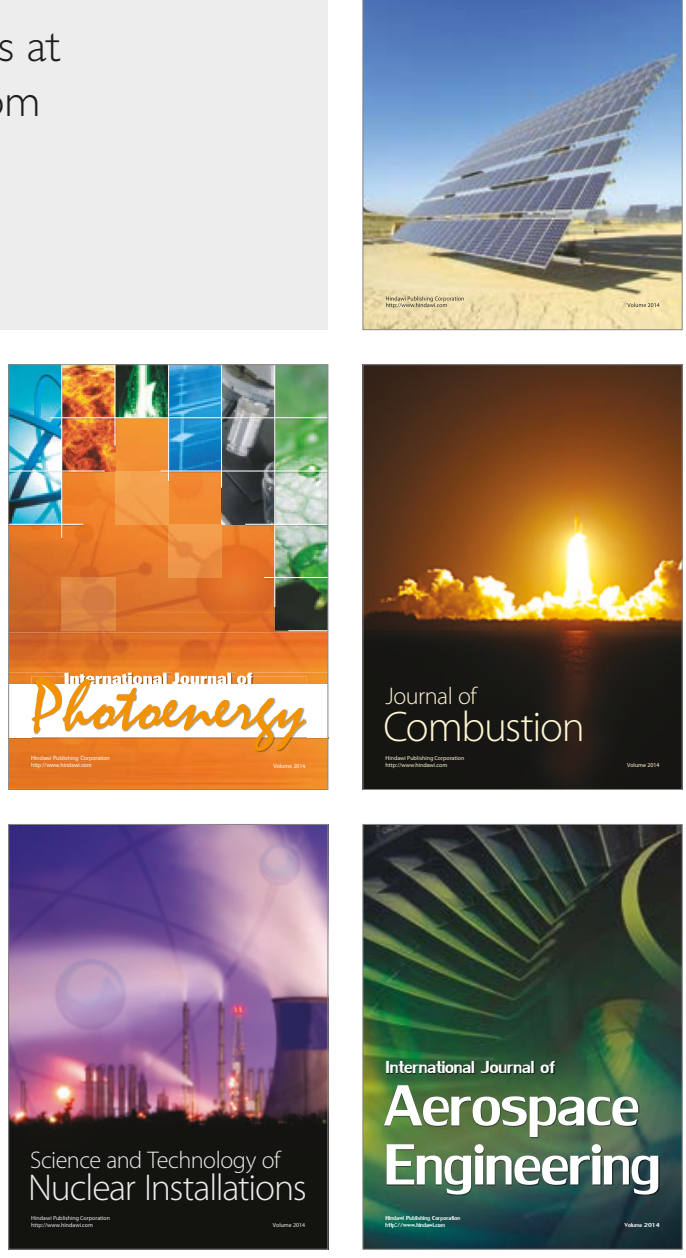